Laser Chem., 1999, Vol. 19, pp. 343-348 Reprints available directly from the publisher Photocopying permitted by license only
(C) 1999 OPA (Overseas Publishers Association) N.V. Published by license under the Harwood Academic Publishers imprint, part of The Gordon and Breach Publishing Group.

\title{
STRUCTURE-REACTIVITY CORRELATIONS OF EXCITED STATES OF PERFLUORINATED COMPOUNDS: A TIME RESOLVED RAMAN STUDY
}

\author{
R. ANANDHI, G. BALAKRISHNAN, P. MOHANDAS \\ and S. UMAPATHY* \\ Department of Inorganic and physical chemistry, Indian Institute of Science, \\ Bangalore-560 012, India
}

(Received 18 September 1997)

This paper reports the $\mathrm{TR}^{3}$ spectral studies on perfluorinated organic systems with the objective to understand the influence of perfluorination on the excited states. We have recorded the $\mathrm{TR}^{3}$ spectra and Raman excitation profiles of the triplet excited states of decafluorobenzophenone and fluoranil. It is found that the influence of perfluorination is more pronounced in the triplet excited state than the ground state and thus leads to enhanced reactivity for perfluorinated compounds through larger structural distortions.

Keywords: Perfluoroeffect; decafluorobenzophenone; fluoranil; resonance Raman; excited states

\section{INTRODUCTION}

Perfluoro compounds have attracted attention due to their unique structure and reactivity, induced by perfluoro effect [1]. In particular, it is well known that the reactivity of photoexcited states of perfluoro systems are orders of magnitude faster than their counterparts, viz., other halogenated and non-halogenated systems. For example, decafluorobenzophenone (DFBP) is 35 times more reactive towards

\footnotetext{
*Corresponding author. Tel.: -91-80-3092595; Fax: -91-80-3316552; e-mail: suma@)hamsadvani.serc.iisc.ernet.in
} 
hydrogen abstraction from its triplet excited state than benzophenone (BP)[2]. This is, in spite of the fact that their properties, such as, electronic configuration, energy, quantum yield, etc., are very similar in the triplet excited state. The triplet excited states of both BP and DFBP are of $n-\pi^{*}$ in nature, and the quantum yield is reported to be close to unity. Further, from a structural point of view, fluorine, by means of its inductive effect and $\pi$-donation, leads to strengthening of both the $\sigma$ and $\pi$ bonds. Interestingly, $\pi$-donation is considerably weaker than normally observed for other halogen systems such as chlorinated and brominated analogs. This paper addresses the influence of perfluoro effect on the structure and reactivity of excited triplet state, using time resolved resonance Raman $\left(\mathrm{TR}^{3}\right)$ spectroscopy. Two examples are presented, viz. DFBP and tetrafluoro-1,4benzoquinone (fluoranil, FA).

\section{EXPERIMENTAL}

The detailed experimental setup and the procedures used for $\mathrm{TR}^{3}$ Spectroscopy have been described previously [3]. Briefly, laser pulses of wavelength $355 \mathrm{~nm}$ and a range of wavelengths between 450 to $550 \mathrm{~nm}$ were used as the pump and probe excitations. The laser pulses were of about $8-10 \mathrm{~ns}$ in temporal width and the energies of about $2.5 \mathrm{~mJ}$ for both pump and probe laser pulses. For Raman Excitation Profile (REP), measurements were carried out with the probe wavelengths at every $10 \mathrm{~nm}$ intervals. The recorded Raman spectra were calibrated using known solvent bands as reference and the spectral resolution is estimated as $5 \mathrm{~cm}^{-1}$. Depolarization ratios of the Raman bands have been measured by using a polarizer in combination with a scrambler. The solutions were circulated at a rate of $10 \mathrm{ml}$ per minute such that each sample volume received only one laser shot, to avoid photo damage during exposure.

\section{RESULTS AND DISCUSSION}

\section{Decafluorobenzophenone}

It is known from flash photolysis studies that ${ }^{3} \mathrm{DFBP}$ has absorption maximum at $490 \mathrm{~nm}$ and it exists in the $\mathrm{ns}-\mu \mathrm{s}$ time scales. Therefore, 
we have recorded the $\mathrm{TR}^{3}$ spectra of ${ }^{3} \mathrm{DFBP}$ by selecting the Raman excitation (probe) wavelength in resonance with the corresponding absorption maximum and the photoexcitation (pump) with $355 \mathrm{~nm}$ laser pulse. Further, to confirm the origin of the above Raman spectra we have also recorded the $\mathrm{TR}^{3}$ spectra of this intermediate, by tuning the probe wavelength along the absorption spectral profile of the triplet excited state, from 450 to $550 \mathrm{~nm}$. After appropriate normalization of Raman intensities with respect to a solvent band, REPs have been plotted [3]. Thus, we attribute the spectra of the transient state in $\mathrm{CCl}_{4}$ to the triplet state of the DFBP on the basis of the following observations. (1) The decay rate constants of all the observed Raman bands are consistent with the literature data on the transient absorption studies for triplet excited state, (2) The REPs of the transient Raman bands follow the absorption spectrum of the triplet excited state, thus confirming the vibrational spectrum observed corresponds to the ${ }^{3}$ DFBP and (3) In a non interacting solvent like $\mathrm{CCl}_{4}$, the only transient likely to be observed in the first few hundreds of nanoseconds is the ${ }^{3} \mathrm{DFBP}$.

$\mathrm{TR}^{3}$ spectra of DFBP in $\mathrm{CCl}_{4}$, has three bands at 1614, 1391 and $1246 \mathrm{~cm}^{-1}$. Our depolarization studies have shown that all of these bands are polarized. In the ground state of DFBP, the intense bands observed at 1690 and $1640 \mathrm{~cm}^{-1}$ correspond to $\mathrm{C}=\mathrm{O}$ and $\mathrm{C}=\mathrm{C}$ stretching modes, whereas in the triplet excited state we observe only one band in the $1600-1700 \mathrm{~cm}^{-1}$ region. By analogy with the assignment of the ${ }^{3} \mathrm{BP}$ spectra, the bands observed at 1614 and $1246 \mathrm{~cm}^{-1}$ for ${ }^{3} \mathrm{DFBP}$ are assigned to the $\mathrm{C}=\mathrm{C}$ and $\mathrm{C}=\mathrm{O}$ stretching respectively. Further, evidence for the $\mathrm{C}=\mathrm{O}$ assignment has also been obtained by low temperature absorption studies. It is known that vibrational progressions observed in the low temperature absorption spectrum corresponds to the vibrational modes with the largest Franck-Condon activity in the excited state. The resonant excited state in our case is the triplet excited state $\left(T_{1}\right)$ where the $n-\pi^{*}$ transition is localized on the $\mathrm{C}=\mathrm{O}$ moiety. Therefore we have recorded the low temperature $(120 \mathrm{~K})$ absorption spectrum of the $S_{0}-T_{1}$ transition [3]. The vibrational spacing is found to be $1250 \mathrm{~cm}^{-1}$ which supports our assignment for the band observed $1246 \mathrm{~cm}^{-1}$ to $\mathrm{C}=\mathrm{O}$ stretching vibration. The band observed around $1391 \mathrm{~cm}^{-1}$ is assigned to $\mathrm{C}-\mathrm{F}$ stretch, in comparison with the ground state of DFBP. 
The structure of BP is reported to be more planar in the triplet excited state than in the ground state. The difference between the experimentally observed $\mathrm{C}=\mathrm{O}$ and $\mathrm{C}=\mathrm{C}$ stretching frequencies of ${ }^{3} \mathrm{BP}$ and ${ }^{3} \mathrm{DFBP}$ suggests that ${ }^{3} \mathrm{DFBP}$ is more non-planar than ${ }^{3} \mathrm{BP}$. That is, the observed decrease for $\mathrm{C}=\mathrm{C}$ stretching frequency is found to be only $36 \mathrm{~cm}^{-1}$ for ${ }^{3} \mathrm{DFBP}$ on going from the ground to the triplet excited state, whereas for BP the decrease is $60 \mathrm{~cm}^{-1}$. This means that the extent of conjugation of the phenyl ring to carbonyl group is reduced in the excited state of DFBP in relation to BP. Thus one can conclude that the benzene rings are more twisted with respect to the $\mathrm{C}-\mathrm{CO}-\mathrm{C}$ plane in the triplet excited state of DFBP than BP. This is also supported by the observation of the CO frequency of DFBP $\left(1246 \mathrm{~cm}^{-1}\right)$ and BP $\left(1222 \mathrm{~cm}^{-1}\right)$. Based on these, the observation of higher frequency for DFBP compared BP, can be attributed to the presence of a twisted structure in DFBP. Further, the perfluorination will influence the carbonyl group and phenyl rings differently. The phenyl rings are affected both by inductive effect and the $\pi$-donation, whereas, the carbonyl group is more likely to be influenced by inductive effect (since twisted rings would not facilitate $\pi$-donation to the carbonyl). Therefore, we attribute the observed increase in reactivity for excited triplet state of DFBP to the highly polarized nature of the carbonyl group [3].

\section{Fluoranil}

FA is known to undergo various photophysical processes upon photoexcitation, resulting in singlet state, triplet state, exciplexes, etc. The Raman scattering of the triplet excited state is expected to be resonance enhanced under the probe wavelength of $485 \mathrm{~nm}$ since the triplet state has a band at $485 \mathrm{~nm}$ with $\varepsilon_{\max }^{T}=7200 \mathrm{M}^{-1} \mathrm{~cm}^{-1}$. Therefore, to record the $\mathrm{TR}^{3}$ spectra of the triplet state, we have used $355 \mathrm{~nm}$ (pump laser) to generate the triplet excited state of FA and $485 \mathrm{~nm}$ (probe laser) to record the Raman scattering. The $\mathrm{TR}^{3}$ spectra of FA contains many Raman bands with varying degrees of intensities $[4,5]$. The $\mathrm{TR}^{3}$ spectra obtained here is attributed to ${ }^{3} \mathrm{FA}$ for the following reasons: (a) the decay rate constant for all the bands are nearly the same and is similar to the ${ }^{3} \mathrm{FA}$ reported from a transient absorption study. (b) REPs of the various bands reproduces the shape 
of the triplet absorption spectrum well and thus confirming that the resonant electronic state investigated here is the triplet excited state and therefore the vibrational spectral bands observed can be ascribed to the triplet excited state of FA. The vibrational assignments of RR spectra of ${ }^{3} \mathrm{FA}$ have been carried out with the help of comparison with $\mathrm{RR}$ spectra of ${ }^{3} \mathrm{CA}$ and ${ }^{3} \mathrm{BQ}[4,5]$. The assignments are further supported by the calculated vibrational frequencies using $a b$ initio Hartree-Fock quantum mechanical methods with the standard 6$31 \mathrm{G}^{*}$ basis set [6].

Thus the observation of higher frequencies for the $\mathrm{C}=\mathrm{O}$ and $\mathrm{C}=\mathrm{C}$ of FA, both in the ground and triplet excited states with respect to BQ, can be ascribed to perfluoro effect. This is consistent with a recently reported theoretical study on ground state fluoranil. In addition, since the vibrational frequency shifts observed for ${ }^{3} \mathrm{FA}$ in comparison with ${ }^{3} \mathrm{BQ}$ is considerably larger than the corresponding shifts observed for ground state $\mathrm{FA}$ and $\mathrm{BQ}$, it is concluded that perfluoro effect is very pronounced in the triplet excited state. In other words, the electron delocalization in the antibonding $\pi^{*}$ orbitals of ${ }^{3} \mathrm{FA}$ is less than that of ${ }^{3} \mathrm{BQ}$. Similarly, the ground to triplet $\mathrm{C}=\mathrm{O}$ stretching frequency shifts of $\mathrm{BQ}(1663-1496=167)$ and FA $(1700-1621=79)$ and also the $\mathrm{C}=\mathrm{C}$ stretching shifts for BQ $(1657-1554=103)$ and FA $(1700-1647=53)$, indicate that the structural distortion in ${ }^{3} \mathrm{FA}$ is less than that observed for ${ }^{3} \mathrm{BQ}$. The fact that there is a uniform reduction in the shifts of about $50 \%(\mathrm{BQ}$ to $\mathrm{FA}$ ) for both the $\mathrm{C}=\mathrm{O}$ and $\mathrm{C}=\mathrm{C}$ frequencies suggests that the perfluoro effect influence is beyond the first carbon to which fluorine is attached, that is extending to the carbonyl groups.

In summary, the effect of perfluorination on DFBP and FA has been studied using $\mathrm{TR}^{3}$ spectroscopy. The ${ }^{3} \mathrm{DFBP}$ is found to be greatly distorted compared to BP. In the case of ${ }^{3} \mathrm{FA}$, the structure seems to be largely different from that of non-fluorinated counter parts i.e., BQ. The detailed analyses of the $\mathrm{TR}^{3}$ spectra of various photochemical intermediates of DFBP [3] and FA [6] will be reported elsewhere.

\section{References}

[1] Chowdhury, S., Grimsrud, E. P. and Heinis, T. (1986). J. Am. Chem. Soc., 108, 3635.

[2] Shoute, C. T. L. and Mittal, J. P. (1993). J. Phys. Chem., 97, 8630. 
[3] Anandhi, R. and Umapathy, S., Manuscript in preparation.

[4] Balakrishnan, G. and Umapathy, S. (1997). J. Chem. Soc. Faraday Trans., 93, 4125.

[5] Balakrishnan, G. and Umapathy, S. (1997). Chem. Phys. Lett., 270, 557.

[6] Balakrishnan, G., Mohandas, P. and Umapathy, S., Manuscript in preparation. 